\title{
AVALIAÇÃO MORFOFUNCIONAL DE TRANSPLANTE AUTÓGENO DE OVÁRIOS ÍNTEGROS E FATIADOS
}

\author{
MORPHOFUNCTIONAL ASSESSMENT OF INTACT AND SLICED OF AUTOLOGOUS OVARIAN \\ TRANSPLANTATION
}

Andy Petroianu, Luiz Ronaldo Alberti, Leonardo de Souza Vasconcellos

\section{RESUMO}

Objetivo: Os objetivos do presente trabalho foram avaliar aspectos morfológicos e endócrinos de transplantes ovarianos autógenos fixados em posição ortotópica, na forma íntegra e fatiada, sem pedículo vascular. Método: Foram utilizados 42 coelhos da raça Nova Zelândia Branca. No Grupo $1(\mathrm{n}=8)$ foi realizada apenas laparotomia e laparorrafia. No Grupo 2A (n=8), transplantou-se o ovário na forma íntegra; no Grupo 2B transplantou-se o ovário na forma fatiada e no Grupo 2C (n=8), transplantou-se de um lado o ovário íntegro, e do outro lado, fatiado. Não houve anastomose vascular. Dosou-se o estradiol, a progesterona, o hormônio folículo-estimulante e o hormônio luteinizante de cada animal no nono mês pós-operatório. Estudaram-se as morfologias macro e microscópica dos ovários, tubas e útero, de todos os animais. Foram utilizados os testes one-way ANOVA, seguido pelo teste de Tukey-Kramer para comparar as dosagens hormonais, considerando $\mathrm{p}<0,05$. Resultados: Os ovários transplantados estavam rodeados de tecido conjuntivo bem vascularizado, mostrando folículos em diferentes estágios de desenvolvimento. A histologia tubária e uterina também foi preservada em ambos os grupos. A dosagem hormonal foi normal em todas as coelhas, indicando que o ovário transplantado estava funcionante do ponto de vista endócrino. Conclusão: O transplante autógeno ortotópico de ovários na forma íntegra ou fatiada, sem preservação do pedículo vascular é viável e preserva as funções endócrinas.

Descritores: Histologia; Testes de função ovariana, Transplante, Coelhos

\section{Instituição:}

Departamentos de Cirurgia e de Propedêutica Complementar da Faculdade de Medicina da Universidade Federal de Minas Gerais

\section{Correspondência:}

Prof. Andy Petroianu

Av. Afonso Pena, 1626 - apto. 1901 - Belo Horizonte / MG - CEP: 30130-005 - Brasil

Tel./Fax: (31) 32747744

E-mail: petroian@medicina.ufmg.br

Recebido em: 12.05 .2008

\section{INTRODUÇÃO}

Muitas pacientes com câncer sofrem perda iatrogênica da função ovariana mesmo sem o ovário estar envolvido na doença, em decorrência de tratamento radioterápico e quimioterápico. ${ }^{1,2} \mathrm{Em}$ outros casos, a ooforectomia é realizada em procedimentos sobre a pelve, mesmo sem haver inconveniência na sua manutenção. Essa conduta resulta em menopausa precoce e distúrbios funcionais, tais como disfunção sexual, níveis alterados de lipoproteínas, além de maior risco de osteoporose e doenças cardíacas. ${ }^{1-4}$

Uma alternativa fisiológica na manutenção da função hormonal em mulheres que precisam se submeter à retirada de ovários normais nas quais a função regulatória do eixo hipotálamo-hipófisário está intacta e na ausência de doença auto-imune é o transplante ovariano. Diversas técnicas experimentais de auto-implante ovariano vêm sendo pesquisadas. ${ }^{4-6}$ A multiplicidade de espécies animais e dos métodos estudados para preservação da função ovariana resulta em informações conflitantes quanto à eficácia da preservação hormonal. . $^{7-9}$

A utilização de tecidos ovarianos criopreservados e reimplantados em posição heterotópica é uma alternativa mais fisiológica de manter a função hormonal no pós-operatório tardio da retirada de ovários normais. ${ }^{6,79-11}$ 
O objetivo do presente trabalho foi avaliar aspectos morfológicos e endócrinos de transplantes ovarianos autógenos fixados em posição ortotópica na forma íntegra e fatiada sem pedículo vascular.

\section{MATERIAIS E MÉTODOS}

Este trabalho foi realizado de acordo com recomendações das Normas Internacionais de Proteção a Animais e do Código Brasileiro de Experimentação Animal (1988), tendo sido aprovado pela Comissão de Ética do Departamento de Cirurgia da Faculdade de Medicina da Universidade Federal de Minas Gerais (UFMG).

Foram estudados 42 coelhos (Oryctogalus cuniculus) da raça Nova Zelândia Branca, sendo 32 fêmeas e dez machos sabidamente férteis. No início do experimento, todos os coelhos estavam com quatro meses de idade, sexualmente maduros e com peso variando de 2450 g a 2700 g. Os animais provenientes do Biotério Central da Escola de Veterinária da UFMG foram mantidos em gaiolas individuais, onde receberam ração e água à vontade. No pré-operatório todos permaneceram em adaptação e observação do estado de saúde por 20 dias. Após esse período, os animais foram mantidos em jejum pré-operatório de 12 horas.

As coelhas foram aleatoriamente distribuídas em:

Grupo 1: $(n=8)$, animais submetidos apenas à laparotomia mediana e laparorrafia.

Grupo 2: transplante autógeno de ambos os ovários

Subgrupos

$$
\begin{aligned}
& 2 A:(n=8) \text { : íntegro bilateral } \\
& \text { 2B: }(n=8) \text { : fatiado bilateral } \\
& \text { 2C: }(n=8) \text { : íntegro de um lado e fatiado do outro } \\
& \text { lado }
\end{aligned}
$$

Todos os animais receberam antibioticoprofilaxia com cefadroxila (Cefamox; Bristol-Myers-Squibb, São Paulo) na dose de 50mg/kg dissolvida em $10 \mathrm{ml}$ de leite e administrada por cateter orogástrico, trinta minutos antes do procedimento cirúrgico.

A anestesia foi induzida com injeção de cloridrato de quetamina (Ketalar ${ }^{\circledR}$, Pfizer, São Paulo) intramuscular na região glútea a $5 \%$, na dose de $50 \mathrm{mg} / \mathrm{kg}$. Quando necessário, foi aplicado adicionalmente um quarto da dose inicial do anestésico. Durante todo o período anestésico, foram observadas as freqüências cardíaca e respiratória, além da movimentação voluntária dos coelhos, visando detectar possíveis complicações.

Após tricotomia do abdome, realizou-se anti-sepsia com solução de polivinilpirrolidona (PVPI $®$ ) degermante, seguida de solução alcoólica de iodo a $2 \%$. A assepsia foi seguida da colocação de campos operatórios.

Os animais do Grupo 1 foram submetidos a laparotomia mediana infraumbilical, identificação do útero, das tubas uterinas e dos ovários. Em seguida, realizou-se laparorrafia com suturas contínuas em dois planos, aponeurose com fio de poliglactina 2-0 (Vicryl $\AA$, Ethicon, São Paulo) e pele com náilon 3-0 (Nylon ${ }^{\circledR}$, Ethicon, São Paulo).

Nos animais do Grupo 2, realizou-se ooforectomia bilateral através de laparotomia mediana infra-umbilical, com cuidado para preservar a integridade das tubas uterinas. Em seguida, os ovários foram reimplantados ortotopicamente sem anastomose vascular e fixados com um ponto de fio de náilon 5-0 (Nylon ${ }^{\circledR}$, Ethicon, São Paulo), de acordo com os seguintes subgrupos: Subgrupo 2A - submetido a transplante autógeno bilateral ortotópico dos ovários íntegros, Subgrupo 2B - os ovários foram cortados longitudinalmente em três fatias de $2 \mathrm{~mm}$ de espessura com bisturi e, em seguida, foram transplantados ortotopicamente, Subgrupo $2 \mathrm{C}$ - transplantaramse os ovários ortotopicamente, de um lado, na forma íntegra, e do outro na fatiada.

Após o ato cirúrgico, as coelhas receberam ração e água previamente filtrada em recipientes próprios, ad libitum e foram mantidas em gaiolas individuais, em condições adequadas de higiene, ventilação e iluminação.

No nono mês após o procedimento cirúrgico, os animais foram novamente anestesiados e submetidos à segunda laparotomia. A veia cava abdominal foi identificada e puncionada para coleta sanguínea por sistema a vácuo (Vacuntainer ${ }^{\circledR}$, Becton Dickinson, Brasil). De cada animal, coletou-se $1 \mathrm{ml}$ de sangue venoso em frasco estéril para soro (tampa vermelha, com gel separador). As amostras sangüíneas coletadas foram imediatamente centrifugadas a $4000 \mathrm{rpm}$ por 10 minutos e submetidas imediatamente a exames laboratoriais. Foram dosados dois hormônios hipofisários, hormônio folículo-estimulante (FSH) e hormônio luteinizante (LH), e dois hormônios gonadais, estradiol e progesterona, pelo método de imunofluorimetria utilizando aparelho de automação AutoDelfia ${ }^{\mathrm{TM}}$ (Wallac Ou, Finlândia). Os valores hormonais obtidos nas coelhas do Grupo 1 (controle) foram considerados como valores de referência para os demais grupos, tendo em vista que não houve estudo hormonal prévio das coelhas operadas.

Após a coleta, todas as coelhas foram mortas por choque hemorrágico, após anestesia profunda com quetamina (100mg/ $\mathrm{kg}$ ). As cavidades torácica e abdominal, bem como seus órgãos foram cuidadosamente estudados. Retirou-se o útero, os ovários e as tubas uterinas de todas as coelhas. Foram obtidos fragmentos de $5 \mu \mathrm{m}$ de cada estrutura, sendo parte deles fixada em solução de Bouin e outra parte em solução de formaldeído a $4 \%$ (formol a $10 \%$ ). Os cortes foram fixados sobre lâminas de vidro e corados com hematoxilina e eosina (HE) para estudo histológico de rotina. A viabilidade ovariana foi avaliada baseada nos seguintes parâmetros histológicos: presença de angiogênese, qualificação dos folículos ovarianos, presença de corpos lúteos.

Foram utilizados os testes one-way ANOVA seguido pelo teste de Tukey-Kramer para comparar as dosagens hormonais (estradiol, progesterona, hormônio folículo-estimulante e hormônio luteinizante). As diferenças foram consideradas significativas para valores correspondentes a $\mathrm{p}<0,05$.

\section{RESULTADOS}

Todos os animais recuperaram-se espontaneamente das cirurgias e sobreviveram durante os nove meses do experimento, sem intercorrências.

A Tabela 1 mostra os valores hormonais obtidos de todas as coelhas. Foram detectados hormônios ovarianos em todas as coelhas submetidas a transplante autógeno. Não houve diferença entre as médias dos valores hormonais nos diversos subgrupos e no Grupo-controle ( $\mathrm{p}>0,05)$.

À segunda laparotomia, a cavidade abdominal do Grupo 1 manteve-se com aspecto normal. Houve aderências entre a tuba e o ovário em duas coelhas. O útero e as tubas não mostraram outras alterações anatômicas. 
Tabela 1. Distribuição dos animais segundo valores hormonais (média e desvio padrão da média) dosados nas coelhas controle e submetidas ao transplante autógeno ortotópico de ovários.

\begin{tabular}{|c|c|c|c|c|}
\hline GRUPOS & $\begin{array}{l}\text { ESTRADIOL } \\
(\mathrm{pg} / \mathrm{ml})\end{array}$ & $\begin{array}{l}\text { PROGESTERONA } \\
(\mathrm{pg} / \mathrm{ml})\end{array}$ & FSH (UI/I) & LH (UI/I) \\
\hline 1 & $2.801 \pm 762$ & $104.200 \pm 2.348$ & $0,13 \pm 0,05$ & $0,16 \pm 0,07$ \\
\hline $2^{a}$ & $2.500 \pm 950$ & $109.300 \pm 5500$ & $0,30 \pm 0,20$ & $0,30 \pm 0,20$ \\
\hline $2 B$ & $2.850 \pm 700$ & $105.400 \pm 3500$ & $0,20 \pm 0,10$ & $0,20 \pm 0,10$ \\
\hline $2 \mathrm{C}$ & $2.950 \pm 850$ & $106.100 \pm 3300$ & $0,30 \pm, 20$ & $0,20 \pm 0,10$ \\
\hline
\end{tabular}

G1 - Grupo controle

G2A - Transplante ovariano autógeno na forma íntegra

G2B - Transplante ovariano autógeno na forma fatiada

G2C - Transplante ovariano autógeno na forma integra de um lado e

fatiada, de outro

FSH: Hormônio folículo-estimulante

LH: Hormônio luteinizante

Ao exame macroscópico verificou-se que os ovários das coelhas do Grupo 2 (transplante autógeno) preservaram sua parte externa, sem fibrose ou reação inflamatória. Todavia, encontrou-se hidrossalpinge em dois animais, além de múltiplas aderências entre ovário, tuba e intestino nas outras duas coelhas. O útero de todos os animais tinha aspecto normal. O restante da cavidade abdominal permaneceu sem alterações. Não houve alterações macroscópicas nos demais órgãos abdominais.

Ao estudo histológico, verificou-se que os ovários tanto dos animais controle quanto dos reimplantados estiveram preservados, não havendo diferenças entre eles. Também não foram encontradas diferenças entre ovários reimplantados íntegros ou fatiados. A viabilidade ovariana foi confirmada pela presença de angiogênese satisfatória, vários folículos em diferentes estágios de maturação, bem como corpos lúteos e cistos foliculares em proporções variáveis. Não foram identificados sinais de isquemia ou necrose.

A histologia tubária das coelhas do Grupo 1 foi normal, com microvilosidades bem desenvolvidas, trofismo preservado e ausência de sinais inflamatórios ou degenerativos. No Grupo 2, foram encontradas hidrossalpinge em quatro coelhas. Os demais animais apresentaram histoarquitetura tubária conservada.

A histoarquitetura uterina de todas as coelhas foi mantida. O endométrio evidenciou diversas fases de proliferação compatíveis com as fases do ciclo estral, indicando adequada produção hormonal.

\section{DISCUSSÃO}

Diversas técnicas experimentais de auto-implante ovariano vêm sendo pesquisadas. ${ }^{12-17}$ A multiplicidade de espécies animais e de métodos estudados para a preservação da função ovariana resulta em informações conflitantes quanto à eficácia da manutenção hormonal. ${ }^{18,19}$ Optou-se pelo emprego de coelha como animal de experimentação a partir da observação de sua boa capacidade reprodutiva e da simplicidade do procedimento cirúrgico sem a necessidade de material especial, considerando também a linha de pesquisa desenvolvida por nós no Departamento de Cirurgia da UFMG referente a transplante de órgãos e tecidos.

Utilizou-se quetamina por suas características de induzir estado de sedação, imobilidade e analgesia acentuada, com efeito de anestesia dissociativa, com larga margem de segurança e fácil controle do plano anestésico.

No presente estudo, não houve diferença nas concentrações hormonais entre o Grupo 1 (controle) e o Grupo 2 (submetidos a cirurgia dos ovários). Do mesmo modo, o estudo microscópico ovariano em ambos os grupos não mostrou evidências que sugerissem hiper ou hipofunção hormonal, como por exemplo, hipotrofia ou hiperplasia endometrial, fato esse comprovado pelas dosagens dos hormônios hipofisários e gonadais, que se mantiveram dentro da faixa de normalidade.

O auto-implante ovariano ortotópico avascular foi um bom método para a completa recuperação das funções ovarianas. O suprimento sangüíneo é um fator importante para manter a vitalidade tanto dos auto-implantes quanto dos transplantes de ovário. Os resultados do presente experimento confirmam que a função ovariana pode ser preservada após castração seguida de implante ovariano sem a realização de anastomose vascular em coelhos. Esse fato também fora constatado previamente em ratos., ${ }^{1,2,20-22}$ A ausência de anastomose vascular não interferiu na vitalidade dos ovários, que foram envolvidos por neoformação vascular adequada. Não foram detectadas complicações decorrentes da falta do restabelecimento vascular cirúrgico, pois nenhum dos animais apresentou sinais de isquemia ovariana. Esses achados contradizem alguns autores, que consideram fundamental a revascularização cirúrgica dos enxertos ovarianos para a manutenção de sua funcionalidade. ${ }^{5,23,24}$ Por outro lado, existem estudos que constataram ser a anastomose do pedículo vascular desnecessária para manutenção da vitalidade e função ovariana. A intensa neovascularização que se forma em torno do ovário parece ser suficiente para conservar sua vitalidade ${ }^{24-26}$ Os elementos responsáveis pela estimulação da angiogênese ainda permanecem desconhecidos. ${ }^{27}$ De acordo com a literatura, o tecido ovariano é uma rica fonte de fatores angiogênicos, que estimulam a rápida migração de células endoteliais em casos de transplante, o que leva ao restabelecimento da circulação sangüínea. ${ }^{28}$ Dissen et al. ${ }^{25}$ implantaram córtex ovariano adjacente à veia jugular, e verificaram a revascularização do enxerto 48 horas após o procedimento. Esse fenômeno foi acompanhado de aumento na expressão de agentes angiogênicos específicos, principalmente do fator de crescimento derivado do endotélio. Esses autores também sugeriram que a secreção de gonadotropinas desempenha um papel significativo na resposta vascular. Dentre as gonadotropinas hipofisárias, o FSH parece ser o mais importante, devido ao estímulo à mitose celular da granulosa e à inibição de sua apoptose. O tratamento de folículos antrais de ratos com doses crescentes de FSH causam supressão da fragmentação do DNA dose-dependente, atingindo a supressão máxima de $60 \% .^{26,27}$ Em decorrência da privação de fatores mitogênicos e tróficos, como as gonadotropinas e os esteróides ovarianos, há lesão por apoptose das células do ovário. A dosagem de FSH manteve-se dentro dos limites da normalidade nos grupos estudados.

Um fator descrito como de importância para a sobrevida e desenvolvimento folicular após retirada cirúrgica dos ovários é a temperatura média de conservação do tecido. Ao contrário de outros modelos de transplante de órgãos, o tecido ovariano à temperatura em torno de $37^{\circ} \mathrm{C}$ mostra melhor conservação do órgão, se comparado ao armazenamento em gelo. É possível que os oócitos primordiais e os folículos primários tenham a mesma sensibilidade a temperaturas baixas, assim como os oócitos e folículos maduros..$^{28,29}$ Durante o ato operatório, tomou-se o cuidado 
de realizar o reimplante dos ovários logo após a ooforectomia, com o intuito de manter a temperatura ovariana mais próxima da fisiológica, minimizando possíveis perdas de células ovarianas.

A avaliação macroscópica e microscópica não mostrou diferenças quanto à preservação ovariana quando implantada na forma íntegra ou fatiada. Por outro lado, em estudo prévio com ratas, os resultados hormonais foram melhores no grupo de reimplante ovariano fatiado. ${ }^{1,2,25,30}$ Contudo, essa diferença não foi verificada na coelha. Cabe, portanto, considerar que o implante ovariano fatiado pode ser mais eficaz do que o íntegro. Diante da falta de uniformidade entre os resultados das duas espécies estudadas por nós e a aparente ausência de subsídios na literatura para essa questão, é fundamental prosseguir nessa linha de pesquisa, em busca da resposta a esse problema.

O período da coleta sangüínea para dosagens hormonais coincidiu com a fase de puerpério em todas as coelhas do Grupo-controle. O mesmo não ocorreu com os animais do Grupo 2, o que explica a maior variação hormonal entre os animais de mesmo subgrupo. A dosagem de FSH nas coelhas estudadas não mostrou diferença com significância estatística entre os grupos 1 e 2. Esse fato mostra que os ovários reimplantados mantiveram a produção hormonal de estradiol dentro da normalidade, já que por mecanismo de retroalimentação, qualquer diminuição da produção de estradiol estimularia a liberação do FSH.
A secreção basal de LH é suprimida pela combinação da progesterona secretada pelo corpo lúteo e do estradiol derivado de folículos antrais. ${ }^{6}$ Como a concentração de LH manteve-se dentro dos limites da normalidade nos animais submetidos ao transplante ovariano autógeno, tal fato comprova adequada produção hormonal nos ovários estudados.

A Tabela 1 mostra que a média dos valores hormonais nos animais submetidos ao reimplante esteve dentro da variação normal para progesterona, estradiol, FSH e LH. Portanto, a viabilidade dos ovários reimplantados foi evidenciada pela presença de hormônios séricos circulantes, o que valida o método utilizado como alternativa para preservação hormonal em coelhas.

\section{CONCLUSÃO}

O transplante autógeno ortotópico de ovários na forma íntegra ou fatiada sem preservação do pedículo vascular é viável e preserva as funções endócrinas.

\section{AGRADECIMENTOS}

Agradecemos ao CNPq e à FAPEMIG pelos auxílios financeiros que permitiram a realização deste trabalho.

\section{ABSTRACT}

Purpose: The purpose of this work was to assess endocrine and histological ovarian aspects of rabbits submitted to bilateral oophorectomy and orthotopic autologous intact and sliced ovarian transplantation without a vascular pedicle. Method: 42 New Zealand White and California rabbits were studied. Ovaries were removed and orthotopically transplanted with no vascular anastomoses: Group 1 ( $\mathrm{n}=8$ ), only laparotomy and laparorrhaphy were performed; Group 2A $(\mathrm{n}=8)$ intact ovaries were re-implanted on both sides; Group 2B $(\mathrm{n}=8)$ both ovaries were sliced and orthotopically re-implanted; Group $2 \mathrm{C}(\mathrm{n}=8)$, an intact ovary was re-implanted on one side, and a sliced ovary was re-implanted on the other side. Estradiol, progesterone, follicle-stimulating hormone and luteinizing hormone levels were assessed nine months after surgery. At the end of the experiment, the morphologic aspect of ovaries, uterus, and tubes were performed. One-way ANOVA and Tukey-Kramer tests compared the hormonal dosages. The $p<0.05$ significance was stated. Results: Ovary implants were surrounded by connective tissue; they were well vascularized and presenting follicles in different stages of development. Hormonal values were normal in all rabbits. Conclusion: Entire or sliced orthotopic autologous ovarian transplantation with no vascular pedicle is viable in rabbits, and effective in preserving normal levels of the ovarian hormone.

Keywords: Histology; Ovarian function tests, Transplantation, Rabbits

\section{REFERÊNCIAS}

1. Marmor D. Fertility after antimitotic treatments. Bull Cancer 1994;84:764-9.

2. Mackie EJ, Radford JA, Shalet SM. Gonadal function following chemotherapy for childhood Hodgkin's disease. Med Pediatr Oncol 1996;27:74-8.

3. Resende CAL, Gobbi H, Geber S. Aspectos embriológicos, Histológicos, Anatômicos e Fisiológicos dos Ovários. In: Coronho V, Petroianu A, Santana EM, Pimenta LG, editors. Tratado de Endocrinologia e Cirurgia Endócrina, 1ed. Rio de Janeiro: Guanabara Koogan; 2001. p. 1060-1095.

4. Simões PM, Machado JÁ. Complicações das cirurgias sobre os ovários. In: Coronho V, Petroianu A, Santana EM, Pimenta LG, editors. Tratado de Endocrinologia e Cirurgia Endócrina, 1ed. Rio de Janeiro: Guanabara Koogan; 2001. p. 1126-8.

5. Corleta HVE, Corleta O, Capp E, Edelweiss MI. Subcutaneous autologous ovarian transplantation in Wistar rats maintains hormone secretion. Fertil Steril 1998;70:16-9.
6. Baber R, Adbella H, Studd F. The premature menopause. Prog Obstet Gynaecol 1991;9:209-26.

7. Seethalakshmi L, Flores C, Carboni AA, Bala R, Diamond DA, Menon M. Cyclosporine, its effects on testicular function and fertility in the prepubertal rat. $\mathrm{J}$ Androl 1990;11:17-24.

8. Ramírez G, Navarte J, Bittle PA, Ayers-Chastain C, Dean SE. Cyclosporine-induced aalterations in the hypothalamic hypophyseal gonadal axis transplant patients. Nephron 1991;58:27-32.

9. Speroff T, Dwason NV, Speroff L, Haber RJ. A risk-benefit analysis of elective bilateral oophorectomy. Am J Obstet Gynecol 1991;164:165-74.

10. Aubard Y, Newton H, Scheffer G, Gosden R. Conservation of the follicular population in irradiated by the cryopreservation and orthotopic autografting ovarian tissue. Eur J Obstet Gynecol Reprod Biol 1998;79:83-7. 
11. Von Eye CH, Corleta O, Capp E, Edelweiss MI. Subcutaneous autologous ovarian transplantation in wistar rats maintains hormone secretion. Fertil Steril 1998;70:16-9.

12. Parks JE, Ruffing NA. Factors affecting low temperature survival of mammalian oocytes. Theriogenology 1992;37:59-72.

13. Lara HE, Hiney JK, Dissen GA, Rivier C, Ojeda SR. Functional recovery of the developing rat ovary after transplantation. Endocrinology 1991;129:1849-60.

14. Cornier E, Sibella P, Chatelet F. Études histologiques et devenir fonctionnel des greffes de trompe et d'ovaire chez la rate. J Gynecol Obstet Biol Reprod 1985;14:567-73.

15. Cooper JE. Ethics and laboratory animals. Vet Rec 1985;116:594-5.

16. Petroianu A Pesquisa experimental. In: Petroianu A. Ética, Moral e Deontologia Médicas.1ed Rio de Janeiro: Guanabara Koogan; 2000.185-90.

17. Callejo J, Jáuregui MT, Valls C, Fernandes ME, Cabré S, Lailla J. Heterotopic ovarian transplantation without vascular pedicle in syngenic Lewis rat. Fertil Steril 1999;72:513-7.

18. Namba Y, Yamamoto M, Atrishima K, Eguchi Y. Suppressive effect of perinatal tests on the differentiation of fetal ovaries transplanted into adult males in the rat. J Anat 1997;191:31-8

19. Weissman A, Gotlieb L, Colgan T, Jurisicova A, Greenblatt EM, Casper RF. Preliminary experience with subcutaneous human ovarian cortex transplantation in the NOD-SCID mouse. Biol Reprod 1999;60:1462-7.

20. Rone JD, Halvorson LM, Goodman AL. Ovarian angiogenesis in rabbits. J Reprod Fertil 1993;97:359-65.

21. Nisolle M, Casanas-Roux F, Qu J, Motta P, Donnez. Histologic and ultrastructura evaluation of fresh and frozen-thawed human ovarian xenografts in nude mice. J. Fertil Steril 2000;74:122-9.
22. Gallagher AC, Chambers TJ, Tobias JH. Distinct effects of ovarian transplantation and exogenous 17B estradiol on cancellous bone of osteopenic ovariectomized rats. Eur J Endocrinol 1995;133:483-8.

23. Esquifino AI, Moreno ML, Agrasal C, Villanúa MA. Effects of cyclosporine on ovarian function in sham-operated and pituitary-grafted young female rats. Soc Exp Biol Med 1995;208:397-403

24. Harp R, Leibach J, Black J, Keldahl C, Karow A. Cryopreservation of murine ovarian tissue. Cryobiology 1994;31:336-43.

25. Dissen GA, Lara HE, Fahrenbach WH, Costa ME, Ojeda SR. Immature rat ovaries become revascularized rapidly after autotransplantation and show gonadotropindependent increase in angiogenic factor gene expression. Endocrinology 1994;134:1146-54.

26. Gallagher AC, Chambers TJ, Tobias JH. Distinct effects of ovarian transplantation and exogenous 17B estradiol on cancellous bone of osteopenic ovariectomized rats. Eur J Endocrinol 1995;133:483-8.

27. Esquifino AI, Moreno ML, Agrasal C, Villanúa MA. Effects of cyclosporine on ovarian function in sham-operated and pituitary-grafted young female rats. Soc Exp Biol Med 1995;208:397-403.

28. Petroianu A, Alberti LR, Vasconcellos LS. Histoarquitetura, função endócrina e taxa de gravidez após auto-implante ovariano ortotópico íntegro e fatiado em coelha. Rev Bras Ginecol Obstet 2004;26:117-23.

29. Chaves DNB, Petroianu A, Alberti LR, Pereira WA. Effects of thalidomide, cyclosporine and diclofenac on skin allograft survival in rats. Transpl Proceed 2004;33:1018-20

30. GORE-LANGTON RE. Cyclosporine differentially affects estrogen and progestin synthesis by rat granulosa cells in vitro. Mol Cell Endocrinol 1988;57:187-98. 